\title{
Local Oscillators Phase Noise Cancellation Methods
}

\author{
${ }^{1}$ R. Subha Sharmini Devi, ${ }^{2}$ S.Anbu Suhantha, ${ }^{3}$ A.Lakshmi \\ ${ }^{1,2,3}$ Lecturer, Einstein College of Engineering, Tirunelveli-627012.
}

\begin{abstract}
Orthogonal frequency division multiplexing (OFDM) is a digital multi-carrier modulation scheme that employs a large number of orthogonal Closely-spaced sub-carriers. Each sub-carrier is modulated with a modulation scheme (QPSK or QAM generally) at a low symbol rate. Each of the sub-carrier is then multiplexed together and this forms the OFDM signal. In practice OFDM signals are generated and detected using the Fast Fourier Transform Algorithm. OFDM is a widely recognized modulation scheme for high data rate communications. However, the plementation of OFDM systems suffers from the phase noise introduced by the local oscillator causes two effects: The common phase error $(C P E)$, and The inter carrier interference (ICI). In this paper we address the problem of phase noise suppression in orthogonal frequency division multiplexing (OFDM) systems.
\end{abstract}

\section{Introduction}

Home wireless applications, such as a wireless network connecting home entertainment systems (TV, DVD, etc),demand very high data rates and nearly flawless transmissions. Orthogonal frequency division multiplexing (OFDM) has been employed in many such applications. One example is the OFDM-based UltraWideband (UWB) technology. Although OFDM displays robustness in case of multipath distortion, by inserting guard intervals between OFDM symbols (e.g., a cyclic prefix), it also has a number of disadvantages. One such disadvantage is the sensitivity to phase noise. Phase noise characteristics are relatively poor with low-cost synthesizers in consumer electronics. In OFDM systems, the phase noise causes a common phase error (CPE) and inter-carrier interferences (ICI). The inter-carrier interference could lead to a bit error floor. The CPE is relatively easy to estimate and compensate, since it identically affects all sub-carriers. However, ICI estimation and cancellation techniques are generally associated with high complexity. In this paper, a low-cost ICI cancellation method is proposed. Significant performance improvements over compensating only the CPE have been achieved. Simulation results are given.

\section{The Phase Noise Estimation Algorithm}

Assuming perfect frequency and timing synchronizations,only phase noise is considered. The $n$th received sample of themth OFDM symbol is

$$
r_{m}(n)=\left\{x_{m}(n) \otimes h_{m}(n)\right\} \cdot e^{j \varphi_{m}(n)}+\xi_{m}(n)(1)
$$

where $x_{m}(n), h_{m}(n)$ and $\varphi_{m}(n)$ represent the transmitted signal, the channel impulse response and the phase noise,respectively. In addition, $\xi_{m}(n)$ is the additive white Gaussian noise. Assuming the cyclic prefix is sufficiently long for OFDM symbols to be ISI-free, the index $m$ does not play any role in the subsequent discussions. Hence, without loss of generality, the subscript $m$ is dropped.

After removing the cyclic-prefix and taking the DFT, the frequency-domain signal is ([2], [3])

$$
R_{k}=X_{k} H_{k} I_{0}+\sum_{i \neq k} X_{l} H_{l} I_{l-k}+\xi_{k}
$$

Where $X_{k}, H_{k}$ and $\xi_{k}$ correspond to the frequency responses of $x(n), h(n)$ and $\xi(n)$, respectively. In the first term of (2), $I_{0}$ causes a phase distortion that is common to all subcarriers within that OFDM symbol. The second term of (2) corresponds to the interferences from adjacent subcarriers. To cancel the CPE and ICI effects, one could seek to estimate the CPE, $I_{0}$, as well as the ICI values, ${ }_{m}$, for $m= \pm 1, \pm 2, \ldots$, from the received symbols, $R_{k}$.

Focusing on the CPE term, $I_{0}$, and the main contributors of ICI, e.g., $I_{-1}$ and $I_{1},(2)$ is modified to

$R_{k}=X_{k} H_{k} I_{0}+X_{k-1} H_{k-1} I_{-1}+X_{k+1} H_{k+1} I_{1}$

$$
+\xi_{\text {res } C l}+\xi_{k}
$$

Where $\xi_{r e s}$ corresponds to the additional noise due to the residual, uncompensated, ICI. 
To estimate $I_{\mathrm{c}}$ from $R_{k}$, one could first multiply both sides of (3) by $X_{k}{ }^{3}{ }_{k}$, , then, add $N$ such products together $(N$ is no greater than the total number of subcarriers). That is,

$$
\begin{aligned}
\sum_{k} R_{k} X_{k}{ }^{*} H_{k}{ }^{*} & =\left(\sum_{k}\left|X_{k} H_{k}\right|^{2}\right) I_{0}+ \\
& +\left(\sum_{k} X_{k-1} H_{k-1} X_{k}{ }^{*} H_{k}{ }^{*}\right) I_{-1} \\
& +\left(\sum_{k} X_{k+1} H_{k+1} X_{k}{ }^{*} H_{k}{ }^{*}\right) I_{1} \\
& +\sum_{k} X_{k}{ }^{*} H_{k}{ }^{*}\left(\xi_{\text {resi } C}+\xi_{k}\right) .
\end{aligned}
$$

Considering that the transmitted data symbols, $X_{k}$, are independent, the term

$$
\left(\sum_{k}\left|X_{k} H_{k}\right|^{2}\right) I_{0}
$$

is expected to be significantly larger than other terms in the right-hand-side of (4), especially when $N$ is very large. This is re-enforced by the observation that the variance of $I_{0}$ is larger than the variances of $I_{1}$ and $I_{-1}$. This is because the power-spectral density (PSD) of typical phase noise exhibits decreasing phase noise power as the frequency moves away from the carrier frequency. Therefore, from (4), $\sum_{k} R_{k} X_{k}{ }^{*} H_{k}{ }^{*} \approx\left(\sum_{k}\left|X_{k} H_{k}\right|^{2}\right) I_{0}$.

Let us define the correlation of the

length $-N$ sequences, $\left\{R_{k}\right\}$ and $\left\{X_{k} H_{k}\right\}$, by $Z_{m}=\sum_{k} R_{k} X_{k+m}{ }^{*} H_{k+m}^{*} \cdot$

$$
\text { In addition, the auto-correlation of }\left\{X_{k} H_{k}\right\} \text { is defined as }
$$

$D_{m}=\sum_{k} X_{k} H_{k} X_{k+m}{ }^{*} H_{k+m}{ }^{*}$.

Thus, according to (5), ${ }^{I_{0}}$ can be obtained as

$$
I_{0}=Z_{0} / D_{0} .
$$

Turning to ICI estimation, to estimate $I_{1}$, one multiplies both sides of (3) by $X_{k+1}{ }^{*} H_{k+1}$, and add $N$ such products, as

$$
\begin{aligned}
\sum_{k} R_{k} X_{k+1}{ }^{\circ} H_{k+1}^{*} & =\left(\sum_{k} X_{k} H_{k} X_{k+1}{ }^{*} H_{k+1}{ }^{\circ}\right) I_{0}+ \\
& +\left(\sum_{k} X_{k-1} H_{k-1} X_{k+1} H_{k+1}\right) I_{-1} \\
& +\left(\sum_{k}\left|X_{k+1} H_{k+1}\right|^{2}\right) I_{1} \\
& +\sum_{k} X_{k+1} H_{k+1}\left(\xi_{\text {ress }}+\xi_{k}\right) .
\end{aligned}
$$

The two terms,

$$
\left(\sum_{k} X_{k} H_{k} X_{k+1}{ }^{*} H_{k+1}{ }^{\circ}\right) I_{0} \text { and }\left(\sum_{k}\left|X_{k+1} H_{k+1}\right|^{2}\right) I_{1}
$$

are expected to dominate the right-hand-side of (9). Hence,

$$
\begin{aligned}
\sum_{k} R_{k} X_{k+1} H_{k+1} & \approx\left(\sum_{k} X_{k} H_{k} X_{k+1} H_{k+1}\right) I_{0}+ \\
& +\left(\sum_{k}\left|X_{k+1} H_{k+1}\right|^{2}\right) I_{1} .
\end{aligned}
$$

In view of the definitions of (6) and (7), $I_{1}$ can be computed as

$$
I_{1}=\left(Z_{1}-I_{0} D_{1}\right) / D_{0} \text {. }
$$

Similarly,

$$
I_{-1}=\left(Z_{-1}-I_{0} D_{1}^{*}\right) / D_{0} .
$$

Once the CPE and ICI terms are estimated, the impairment correction can be accomplished by removing the interference terms, as $\hat{R}_{k}=\left(R_{k}-X_{k-1} H_{k-1} I_{-1}-X_{k+1} H_{k+1} I_{1}\right) / I_{0}$ 
Where $X_{k}$ values correspond to the transmitted QAM symbols. The next section discusses how these $X_{k}$ values might be obtained.

\section{A. CPE Estimatoin using Pilots}

\section{Pilots And Symbol Decisions}

The CPE and ICI estimations, (8), (11) and (12), require known transmitted symbol values. These may be obtained by using the pilots, as well as using the decision feedback. Before the CPE correction, the received symbols have such large phase errors that no reliable decisions can be made. Hence, initially, the CPE estimation can only be obtained by using the pilots. If such obtained precision does not satisfy the requirement, when only pilots are used, a two-step CPE estimation may be facilitated. The first step uses the pilots to obtain a "coarse" CPE estimate. Sliced symbol values are obtained after the initial compensation. The next step uses these sliced values to obtain a fine-tuned CPE estimation. In UWB-OFDM, there are 12 pilot tones in each OFDM symbol. Simulations show that the accuracy of the CPE estimation using only the pilots is satisfactory. After compensating using the estimated CPE, as

$$
R_{k}^{\prime}=R_{k} / I_{0}
$$

only ICI remains. Hence,

$$
R_{k}^{\prime}=X_{k} H_{k}+\sum_{l \neq k} X_{l} H_{l} I_{k-l}^{\prime}+\xi_{k}^{\prime}
$$

Where

$$
I_{k}^{\prime}=I_{k} / I_{0}, \xi_{k}^{\prime}=\xi_{k} / I_{0} .
$$

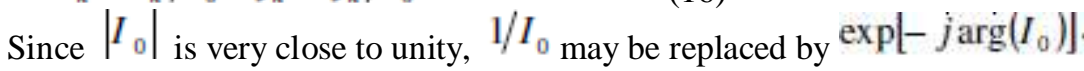

It follows that, using the CPE-compensated $R_{k}^{\prime}$

to compute $D_{0}, D_{0}, Z_{1}$ and $Z_{-1}$, according to (6) and (7), the estimated ICI values are

$$
\begin{aligned}
& I_{1}=\left(Z_{1}-D_{1}\right) / D_{0} \\
& I_{-1}=\left(Z_{-1}-D_{1}^{*}\right) / D_{0} .
\end{aligned}
$$

\section{B. ICI Estimation using Decision Feedback}

In (17) and (18), the computation of $D_{0}, D_{1}, Z_{1}$ and $Z_{-1}$ require known transmitted symbols values. In addition to the known pilots, symbol decisions must be used. To minimize performance degradation due to decision errors, a technique is employed to determine the reliability of the symbol decisions.

Two criteria are used. First, the squared-magnitude of the channel coefficient must be above a threshold. Secondly, the distance between the equalized, received value and its nearest constellation point must be within a threshold. Those symbols that fail to satisfy either of the criteria are declared as erasers.

Let us use the following example - the computation of $I_{1}-$ to demonstrate the required modifications to (6) and (7) in regard to the erasers. Defining $L$ as a set of integers containing the indices of all unreliable decisions, and denoting $H_{k} X_{k}$ by $Y_{k}$, the values, $Z_{1}, D_{0}$ and $D_{1}$ shall be modified to

$$
\begin{aligned}
& Z_{1}=\sum_{\substack{k \neq L \\
k-1 \in L}} R_{k+1} Y_{k}^{*} \\
& D_{0,1}=\sum_{\substack{k \in L \\
k-l \neq L}}\left|Y_{k}\right|^{2} \\
& D_{1}=\sum_{\substack{k \in L \\
k-k}} Y_{k+1} Y_{k}^{*} .
\end{aligned}
$$

Next, $I_{1}$ is calculated as

$$
I_{1}=\left(Z_{1}-D_{1}\right) / D_{0,1}
$$

In the compensation step, as in (13), for a tone that is declared as an eraser, the average value between its two nearest constellation points is used as $X_{k}$. 


\section{Cost Reduction}

It is obvious that hardware savings can be achieved by reducing $N$, the number of terms in the sum in (6) and (7). There are two considerations when choosing the tones to be used to estimate ICI.

First, consecutive tones are needed to estimate the ICI terms. Secondly, it is desirable to use "good" tones (tones that have higher gains in a frequency selective channel) to maximize the signal-to-noise ratio in the estimated ICI. Once the value of $N$ is chosen, the best $N$ consecutive tones can be selected using the following method:

During initial channel estimate, the $N$ consecutive tones having the largest total gain are determined by using a sliding window of length $N$. One could slide this window over all pilot and data tones, and calculate the total power of those tones in the length $N$ window. The group of $N$ consecutive tones having the largest total power will be selected to estimate the ICI.

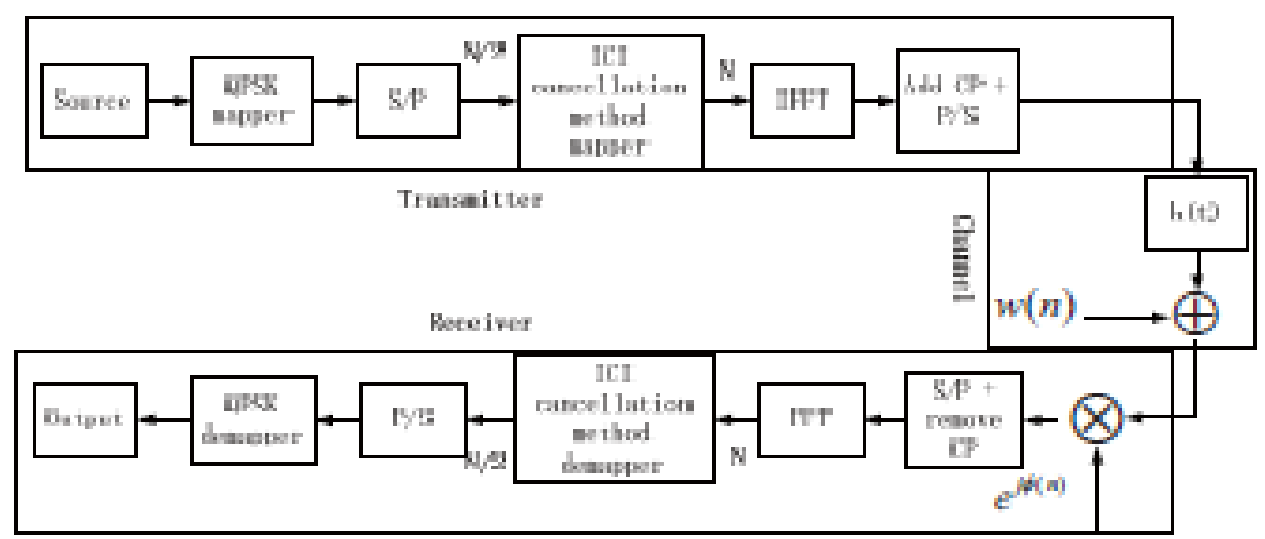

Fig.1 Diagram of OFDM system using the proposed method

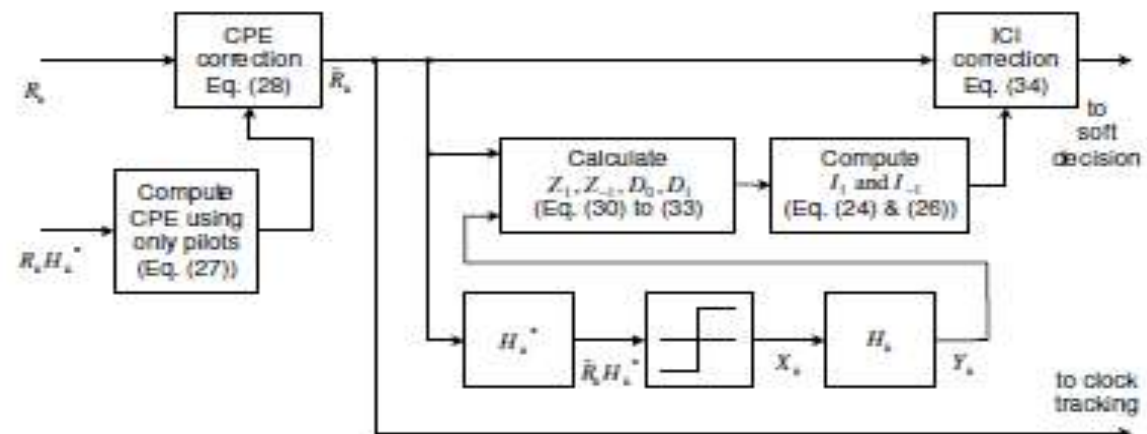

Fig. 2. Details of the CPE and ICI compensation in Fig. 1.

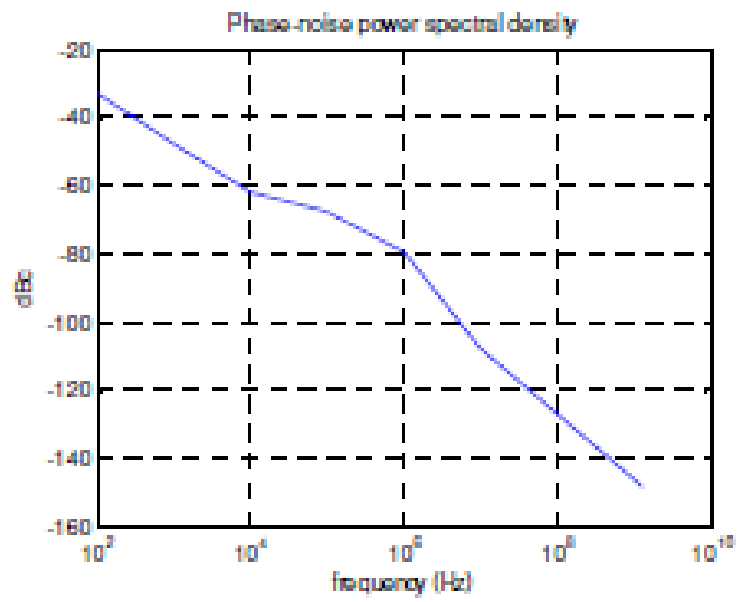

Fig. 3. Phase noise model in the simulation 


\section{Numerical Results}

To evaluate the performance of the proposed method, the UWB-OFDM system is used. The data rate is $480 \mathrm{mbps}$, where the dual-carrier modulation (DCM) is employed. Block diagram of the simulation model of the modem is shown in Fig. 1.

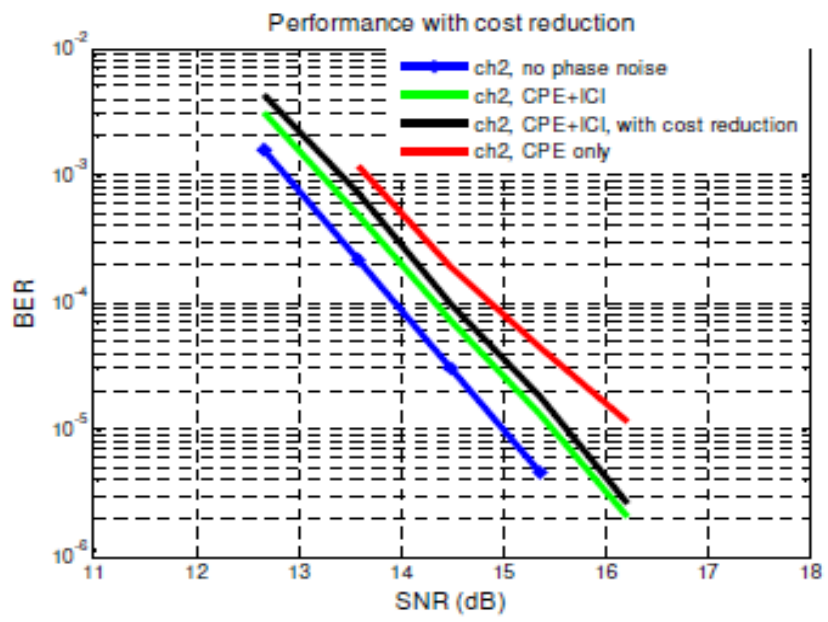

Fig. 4. BER performance with cost reduction.

In the impairment model, the channels are generated according to the CM3 channel power delay profiles. The onesided phase noise PSD is illustrated in Fig 3. Its characteristics correspond to a particular low-cost synthesizer. The impairment model also includes a carrier frequency offset and a clock drift.

In Fig. 1, the initial channel estimation and carrier offset estimation are performed using a preamble (known training symbols). The carrier offset compensation is performed in the time domain. After FFT, CPE and ICI estimations are performed. Since CPE and ICI are uncorrelated from symbol to symbol, the estimated CPE and ICI are applied to the same symbol for phase-noise cancellation - a feed forward correction. The residual carrier frequency offset is also

cancelled by this feed forward-compensation block. The CPE and-ICI-compensated symbols are then used to estimate clock drift and to track channel. Details of the CPE and ICI compensation are provided in Fig. 2.

Fig. 3 shows the BER performance of the Fig. 1 system. Two instances of the CM3 channel (ch1 and ch2 ) are used. For each channel instance, the BER results corresponding to correcting only the CPE, as well as correcting both the CPE and ICI $\left(I_{0}, I_{1}\right.$ and $\left.I_{-1}\right)$, are shown. CPE is estimated using only the 12 pilot tones. ICI estimation uses all data and pilot tones. To demonstrate the effectiveness of the CPE and ICI cancellation, BER results from simulations having no phase noise are also shown. As observed from Fig. 4, at a target BER of $10^{-5}$, when compensating only the CPE, it requires more than $1 \mathrm{~dB}$ higher SNR than the phase-noise-free case. When the ICI, ${ }_{-1}$, and $I_{1}$, are also corrected, it would require just $0.5 \mathrm{~dB}$ higher SNR than the phase-noise-free case. Hence, an improvement of over $0.5 \mathrm{~dB}$ is achieved when ICI cancellation is employed.

Fig. 4 displays results with cost reduction. BER curve obtained by using only 32 tones for ICI estimation is displayed. There is only slight performance degradation as compared with ICI estimation using all tones, while the required computations are much less.

\section{Conclusion}

OFDM has been attractive to future high rate wireless communications. However, the presence of phase noise saturates its performance. Phase noise rise gives rise to common phase error as well as intercarrier interference, and leads to significant performance loss.

Using a relatively low cost CPE and ICI estimator, performance improvement of more than $0.5 \mathrm{~dB}$ has been achieved, as compared with compensating only the CPE.

\section{Acknowledgment}

The authors would like to thank teachers and classmates for the fruitful discussion at our Communication Systems Research Laboratory. 


\section{References}

[1] P. Robertson and S. Kaiser, "Analysis of the effects of phase noise in orthogonal frequency division multiplexing (OFDM) systems," in Proc. ICC'95, pp. 1652-1657, 1995.

[2] S. Wu and Y. Bar-Ness, "Computationally efficient phase noise cancellation technique in OFDM systems with phase noise," in Proc. ISSSTA'04, pp. 788-792, 2004.

[3] S. Wu and Y. Bar-Ness, "A new phase noise mitigation method in OFDM systems with simultaneous CPE and ICI correction," in Proc.MCSS’03, Sept, 2003.

[4] J. A. C. Bingham, "Multicarrier modulation for data transmission: an idea whose time has come," IEEE Communications Magazine, vol. 28,no. 5, pp. 5-14, May 1990.

[5] R. D. J. Van Nee and R. Prasad, OFDM for Wireless Multimedia Communications: Artech House, Incorporated, Jan. 2000.

[6] B. Le Floch, R. Halbert-Lasalle, and D. Castellain, "Digital audio broadcasting to mobile receivers," IEEE Trans. Commun. Electron, vol.35, no.3, pp.493-503, Aug.1989.

[7] H. Sari, G. Karam, and J. Janclaude, "Transmission techniques for digital terrestrial TV broadcasting," IEEE Trans. Commun. Mag,vol.36, pp.100-109, Feb.1995.

[8] T. Pollet, M. V. Bladel, and M. Moeneclaey, "BER sensitivity of OFDM systems to carrier frequency offset and Wiener phase noise,"IEEE Trans. on Commun., vol. 43, no. 2, pp. 191-193, Feb. 1995.

[9] T. Tomba, "On the effect of wiener phase noise in OFDM systems," IEEE Transactions on Communications, vol. 46, no. 5, pp. 580-583,1998.

[10] P. Robertson and S. Kaiser, "Analysis of the effects of phase-noise in Orthogonal Frequency Division Multiplex (OFDM) systems," in Proc.IEEE Int. Conf. Commun. (ICC), Jun. 1995, pp. 1652-1657. 